\title{
Validação psicométrica do general comfort questionnaire em renais crônicos hemodialíticos
}

\section{Psychometric validation of the general comfort questionnaire in chronic patients under kidney hemodialysis Validación psicométrica del General Comfort Questionnaire en pacientes renales crónicos en hemodiálisis}

Geórgia Alcântara Alencar Melo ${ }^{1}$ io https://orcid.org/0000-0002-3886-5646

Renan Alves Silva ${ }^{1}$ id https://orcid.org/0000-0002-6354-2785

Francisco Gilberto Pereira' ${ }^{2,3}$ id https://orcid.org/0000-0002-7744-6030

Letícia Aguiar Lima1 id https://orcid.org/0000-0003-0269-1346

Thereza Moreira Magalhães² io https://orcid.org/0000-0003-1424-0649

Viviane Martins da Silva ${ }^{1}$ io https://orcid.org/0000-0002-8033-8831

Joselany Áfio Caetano ${ }^{1}$ io hitps://orcid.org/0000-0002-0807-056X

\section{Como citar:}

Melo GA, Silva RA, Pereira FG, Lima LA, Magalhães TM, Silva VM, et al. Validação psicométrica do general comfort questionnaire em renais crônicos hemodialíticos. Acta Paul Enferm. 2020;33:eAPE20190258.

DOI

http://dx.doi.org/10.37689/actaape/2020A002585

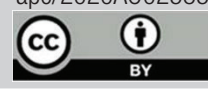

Conforto do paciente; Doença renal crônica; Teoria de enfermagem; Confiabilidade e validade; Análise

fatorial

Keywords

Patient confort: Renal insufficiency, chronic; Nursing theory; Reproducibility of results; Factor analysis, statistical

Descriptores

Comodidad del paciente; Insuficiencia rena crónica: Teoría de enfermeira; Reproducibilidad de Ios resultados; Análisis factorial

\section{Submetido \\ 18 de Setembro de 2019 \\ Aceito \\ 16 de Março de 2020}

Autor correspondente

Renan Alves Silva

E-mail: renan.dehon@gmail.com

\section{Resumo}

Objetivo: Avaliar as propriedades psicométricas do General Comfort Questionnaire, versão Brasileira.

Métodos: Estudo metodológico. Participaram 260 pacientes renais crônicos submetidos a aplicação do General Comfort Questionnaire, versão Brasileira, submetido a análise fatorial exploratória e confiabilidade de dados.

Resultados: a amostra foi considerada adequada pelo teste de Kaiser-Meyer-Olkin $(0,815 ; p<0,001)$. Na análise exploratória de fatores pelo método de estimação dos componentes principais foram obtidos 10 fatores que explicaram 60,14\% da variabilidade da medida. Decidiu-se utilizar o teste do scree plot resultou em quatro fatores (psicoespiritual; sociocultural; ambiental e físico) que explicaram 38,01\% da variância total. 0 valor de alfa de Cronbach geral dos 48 itens foi de 0,83 , com a exclusão dos itens com baixa comunalidade identificou-se alfa de Cronbach de 0,80. Com isso, constata-se que permaneceram 33 itens entre as versões validadas entre 0 grupo de especialistas e a análise fatorial exploratória respeitando os princípios psicométricos com perda de 15 itens.

Conclusão: 0 QCG é válido e confiável para medir o conforto em pacientes renais crônicos em tratamento hemodialítico.

\section{Abstract}

Objective: To assess the psychometric properties of the General Comfort Questionnaire, Brazilian version.

Methods: A methodological study. Participants were 260 chronic patients under kidney hemodialysis submitted to application of the General Comfort Questionnaire, and submitted to exploratory factor analysis and data reliability.

Results: the sample was considered adequate by the Kaiser-Meyer-Olkin test $(0.815 ; p<0.001)$. In the exploratory analysis of factors by estimating the main components, 10 factors were obtained, which explained $60.14 \%$ of the measure variability. The scree plot test use resulted in four factors (psychospiritual, sociocultural, environmental, and physical) that explained $38.01 \%$ of the total variance. The overall Cronbach's Alpha value of the 48 items was 0.83 . Excluding items with low commonality, Cronbach's Alpha of 0.80 was identified. Thus, it was observed that 33 items remained among the validated versions between the group of experts and the exploratory factor analysis, respecting the psychometric principles, with loss of 15 items.

Conclusion: GCQ is valid and reliable for measuring comfort in chronic patients under kidney hemodialysis. 


\section{Resumen}

Objetivo: Evaluar las propiedades psicométricas del General Comfort Questionnaire, versión brasileña.

Métodos: Estudio metodológico. Participaron 260 pacientes renales crónicos, a quienes se les aplicó el General Comfort Questionnaire, versión brasileña, sometido al análisis factorial exploratorio y confiabilidad de datos.

Resultados: La nuestra fue considerada adecuada a través de la prueba Kaiser-Meyer-Olkin $(0,815 ; p<0,001)$. En el análisis exploratorio de factores mediante el método de estimación de los componentes principales, se obtuvieron 10 factores que evidenciaron el 60,14 \% de la variabilidad de la medida. Se decidió utilizar el test scree plot que tuvo como resultado cuatro factores (piscoespiritual, sociocultural, ambiental y físico), que evidenciaron el 38,01 \% de la varianza total. El valor del alfa de Cronbach general de los 48 ítems fue de 0,83, excepto los ítems de baja comunalidad en los que se identificó alfa de Cronbach de 0,80. De esta forma, se verifica que permanecieron 33 ítems entre las versiones validadas por el grupo de especialistas y el análisis factorial exploratorio, respetando los principios psicométricos con una pérdida de 15 ítems.

Conclusión: el GCQ es válido y confiable para medir el confort de pacientes renales crónicos bajo tratamiento hemodialítico.

\section{Introdução}

O conforto possui constructo de aspecto individual e subjetivo que perpassa por aspectos físicos, ambientais, socioculturais e psicoespirituais. É uma dimensão do cuidado próprio da enfermagem dada sua natureza holística; e pode ocorrer em maior ou menor medida dependendo de diferentes fatores, envolvendo os indivíduos e suas percepçóes pessoais. ${ }^{(1)}$ É direcionado a todas pessoas em qualquer etapa do seu ciclo vital. No entanto, este conceito ganha particular interesse em situaçóes de comprometimento da saúde ou vulnerabilidade social, dado sua abrangência e aplicabilidade.

A teórica Katherine Kolcaba define conforto como o estado no qual as necessidades de alívio, tranquilidade e transcendência são fortalecidas nos quatro contextos da experiência humana: físico, psicoespiritual, sociocultural e ambiental, e significa o resultado da ajuda, como vivência subjetiva do estado momentâneo em que a pessoa se percebe tranquila, aliviada ou capaz de superar o desconforto. (2) Com base nessa definição Kolcaba construiu o General Comfort Questionnaire (GCQ), instrumento com 48 itens que contemplam os quatro contextos do conforto, genérico, autoaplicável e capaz de identificar aspectos positivos e negativos envolvidos na prestação de cuidados a um paciente independente da sua condição de saúde. ${ }^{(2)}$

O GCQ já foi utilizado em diversos contextos e países. ${ }^{(3-5)}$ No Brasil, o Questionário de Conforto Geral (QCG) foi traduzido e adaptado seguindo as etapas de Beaton; ${ }^{(6)}$ bem como foi validado seu conteúdo por especialistas na temática. ${ }^{(7)}$ No entanto, para que o questionário seja válido e confiável, torna-se fundamental avaliar a estrutura fatorial e a invariância de medida desse instrumento. $\mathrm{O}$ estudo assume-se de importância decisiva dado o elevado potencial do QCG para mensuração do nível de conforto de pacientes. Aceitando o instrumento como referencial para aferir esse constructo, faz-se necessário que suas propriedades psicométricas sejam avaliadas.

Corroborando com estudos recentes, salienta-se que pacientes renais crônicos hemodialíticos vivenciam falta de alívio, tranquilidade e transcendência nas dimensóes física, psicoespiritual, ambiental, cultural e/ou social por meio de diversas manifestaçôes, entre elas: alteração no padrão de sono, ansiedade, choro, desconforto e descontentamento com a situação, incapacidade de relaxar, inquietação, irritabilidade, lamento, medo, prurido, sensação de calor, sensação de fome, sensação de frio, sintomas de sofrimento e suspiros. ${ }^{(8,9)}$

Desse modo, o objetivo do estudo foi avaliar as propriedades psicométricas do Questionário de Conforto Geral - Versão Brasileira.

\section{Métodos}

Trata-se de um estudo metodológico, transversal, com abordagem quantitativa. A pesquisa foi realizada em três clínicas de hemodiálise, que atendem o maior número de pacientes renais crônicos da região metropolitana de Fortaleza.

Fizeram parte desse estudo 260 pacientes das clínicas com tempo de hemodiálise de no mínimo 12 meses que concordaram em participar do estudo e assinaram o termo de consentimento livre e 
esclarecido, possuíssem idade superior a 18 anos, com pontuação na escala de Coma de Glasgow igual a 15 , e que apresentasse acuidade auditiva e visual preservada. Definiu-se como critério mínimo temporal em virtude do processo de adaptação ou ajustamento as condiçóes clínicas terapêuticas, que poderiam enviesar as observaçóes encontradas. Foram excluídos os pacientes que estavam sob efeito de ansiolíticos ou antidepressivos no período de até 24 horas anteriores à aplicação do instrumento.

$\mathrm{O}$ período de coleta foi realizado em agosto de 2017 a março de 2018. Os instrumentos foram aplicados por meio de entrevista durante a terapia dialítica nos 3 turnos, manhã e tarde e noite, conforme o funcionamento da unidade de hemodiálise. O tempo médio de aplicaçáo dos dois instrumentos para cada participante foi de 20 a 30 minutos.

Para a coleta de dados foram utilizados dois instrumentos de coleta de dados, um para caracterização da amostra com questóes sobre os dados sóciodemográficos e clínicos, e o General Comfort Questionnaire (GCQ) versão traduzida e adaptada para uso no Brasil em pacientes renais crônicos hemodialítico.

O GCQ é estruturado em quatros domínios: físico, sociocultural, ambiental e espiritual. Foi validado quanto a pertinência, clareza e relação com o conforto, possui alfa de Cronbach de 0,80 na seção pré-teste, indicando boa adequação do questionário e uma ótima consistência interna dos itens. Esse instrumento possui ponto de corte maior ou igual a 152 pontos para o estabelecimento de conforto, com valores mínimo e máximo de 48 e 192, respectivamente. ${ }^{(6)}$

A validade de construto do QGC foi avaliada inicialmente pela Análise Fatorial Exploratória (AFE) para identificação de um novo modelo estrutural, pelo método dos componentes principais com rotação Varimax (rotação ortogonal fatores não correlacionados) e eigenvalue superior a $1 . \mathrm{O}$ eigenvalue avalia a contribuição do fator ao modelo construído pela análise fatorial, sendo que valor inferior a 1 sugere pequena contribuição do fator na explicação das variaçóes das variáveis originais. ${ }^{(10)}$ Produziu-se, para cada item, a carga fatorial (saturação) no fator, que indica a correlação entre o item e o fator, de tal forma que quanto mais próximo de
100\% de covariância, melhor é considerado o item, uma vez que representa fortemente o traço latente medido pelo fator. Portanto, a descrição dos fatores em termos dos itens que o constituem é feita com base na magnitude das correlaçôes. ${ }^{(10)}$

Um método alternativo recomendado por especialistas em análise fatorial é o scree plot, que consiste em posicionar um gráfico eigenvalues contra um número de itens presentes. ${ }^{(11)}$

O número de fatores é selecionado por meio da observação de uma ruptura ou descontinuidade entre os valores mais elevados e mais baixos de eigenvalues. Os pontos acima da descontinuidade correspondem ao número de fatores da medida. Para determinar onde ocorre ruptura, traça-se uma linha reta através dos valores mais baixos do traçado de eigenvalues. ${ }^{(11)}$

Foi considerada carga fatorial mínima $=0,40$, para que o item pudesse ser considerado um representante útil do fator. ${ }^{(12,13)}$ Para avaliação da comunalidade, ou seja, o quanto da variância de cada item é explicado por cada fator gerado na análise fatorial, considerou-se satisfatório valor de comunalidade $>0,40{ }^{(12)}$ Valores de comunalidade inferiores nos fatores sugerem uma contribuição pequena do item ao modelo construído ${ }^{(13,14)}$ devendo ser excluído os itens do instrumento.

Empregou-se o Coeficiente Alfa de Cronbach para avaliar a confiabilidade do QCG (escore total e domínios), segundo o critério da homogeneidade dos itens, com valor $>0,80 .^{(11-14)}$

O estudo foi aprovado pelo Comitê de Ética em Pesquisa da Universidade Federal do Ceará, sob parecer 1.482596 .

\section{Resultados}

Identificou-se que a maioria dos participantes estava na faixa etária de 40 a 59 anos (41,7\%), com média de 53,9 anos, do sexo masculino (54,4\%), da cor parda $132(73,3 \%)$, católicos $(68,7 \%)$, casados $(52,8 \%)$, com oito ou menos anos de estudo $(61,4 \%)$. Quanto aos dados clínicos, a maioria era hipertenso $(38,4 \%)$ ou associada a diabetes mellitus $(17 \%)$, que dialisavam por meio da fístula ar- 
teriovenosa (FAV) (68\%) e estavam em tratamento dialítico em até três anos (57\%). O tempo médio de tratamento dialítico foi de 4,85 anos, com tempo mínimo e máximo de dez meses e 21 anos, respectivamente.

$\mathrm{Na}$ análise fatorial exploratória, o teste de esferecidade de Bartlett rejeitou a hipótese nula de que a matriz de correlação dos dados fosse uma matriz identidade $\left(\mathrm{p}<0,001, \mathrm{X}^{2}=2473,826, \mathrm{gl}=528\right)$, e o índice de Kaiser-Meyer-Olkin (KMO) foi de 0,815. Esses resultados demonstraram uma boa adequação da matriz dos dados à análise fatorial, indicando que a análise dos componentes principais poderia ser realizada.

Subsequentemente, optou-se pela análise Exploratória de Fatores para identificação de um novo modelo estrutural para o QGC. Pelo método de estimação dos componentes principais de análise com rotação Varimax e eigenvalues acima de 1 para extração de fatores e carga fatorial superior a 0,40 para seleção dos itens, foram obtidos 10 fatores, os quais, em conjunto, explicaram $60,14 \%$ da variabilidade da medida.

No entanto, o uso de autovalores pode superestimar o número de fatores ${ }^{(11)}$, o que tende a representar um problema em grandes conjuntos de dados, uma vez que produz fatores triviais com poucas variáveis. ${ }^{(14)}$

Assim, no presente estudo, pela observação do scree plot, a linha reta começa a se formar a partir do número 4, sendo constatados, acima deste ponto, somente quatro fatores que explicaram a maior variância $(38,01 \%)$ da medida do QGC, como mostra a figura 1 .

A análise dos componentes principais pelo teste do scree plot resultou em quatro fatores que explicaram 38,01\% da variância total, sendo que cada um mostrou autovalores maiores do que $1(6,04$, $3,19,1,77$ e 1,52 ) e que explicaram $18,30 \%, 9,68 \%$, $5,38 \%$ e 4,63\% da variância da escala, respectivamente. $\mathrm{O}$ quadro 1 apresenta as cargas fatoriais por ordem do item no fator e a percentagem de variância da medida explicada por cada fator, considerando o número de fatores identificados no teste scree plot.

Constatou-se carga fatorial inferior ao desejado nos seguintes itens no fator 1: Item 38, Item 33,

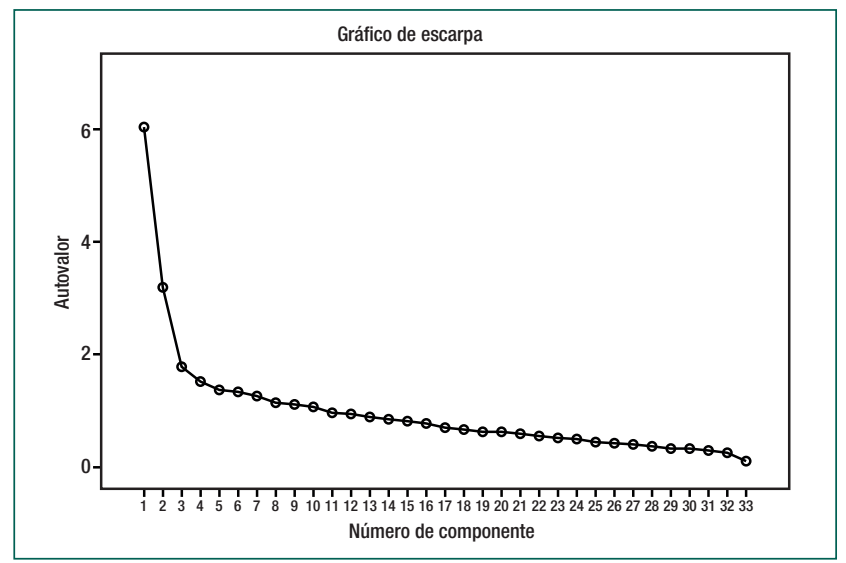

Figura 1. Scree plot de autovalores para os 14 itens do Instrumento do Questionário de Conforto Geral, utilizando-se 0 componente principal de análise

Item 47, Item 4, Item 20, Item 41; fator 2: Item 19; fator 3: Item 42, Item 27, Item 3 e Item 25; fator 4: Item 36 e Item 5. Destaca-se que a análise do conforto agrupados nos fatores possibilitou a seguinte denominação: Fator 1 - Psicoespiritual; Fator 2 Sociocultural; Fator 3 - Ambiental e Fator 4: Físico.

$\mathrm{O}$ valor de comunalidade para cada um dos itens do QGC, que pode ser interpretado como a percentagem da variância de uma variável original explicada pelo número de fatores, é apresentado na Tabela. Constatou-se comunalidade inferior ao desejado nos itens $28,21,6,23,24,44,46,32,16$, $22,31,15,37,13,35,40,39,30,36,12,17,29$, $26,7,43,34,14,48,1,2,47,33,3,4,38,27$, 20, 19, 25, 5, 41. No entanto, decidiu-se excluir os itens que apresentaram comunalidade inferior nos fatores da matriz rotacional.

\section{Análise da confiabilidade}

Os dados evidenciam valores de alfa satisfatórios para o QGC total e em todos os itens. Ainda, verifica-se que caso seja excluído o item 12 do QCC, o valor do alfa de Cronbach aumenta para 0,834 (Quadro 2).

Quando excluídos os itens da análise fatorial com valores de comunalidade inferior a 0,40 nos fatores, evidenciaram valores de alfa satisfatórios para o QGC total $(0,805)$ e fatores, exceto para os itens do fator 3 (ambiental) com 0,576 e 4 (físico) com 0,327 , que não apresentaram correlação item-total alto, gerando valor de alfa inferior ao desejado. Em relação as correlaçóes item-total do fator 3 notou-se que variou entre $-0,366$ a 0,456 e no fator 4 variou 
Quadro 1. Cargas fatorais e valores de comunalidade obtidas pela análise fatorial exploratória do Instrumento do Questionário de Conforto Geral, utilizando-se o componente principal de análise

\begin{tabular}{|c|c|c|c|c|c|}
\hline \multicolumn{6}{|c|}{ Matriz de componente rotativa ${ }^{a}$} \\
\hline & \multicolumn{4}{|c|}{ Componente } & \multirow[b]{2}{*}{$\mathrm{H}^{2}$} \\
\hline & 1 & 2 & 3 & 4 & \\
\hline Item 9 & 0,689 & & & & 0,511 \\
\hline Item 10 & 0,673 & & & & 0,538 \\
\hline Item 23 & 0,540 & & & & 0,381 \\
\hline Item 15 & 0,531 & & & & 0,333 \\
\hline Item 44 & 0,530 & & & & 0,364 \\
\hline Item 17 & 0,511 & & & & 0,279 \\
\hline Item 29 & 0,505 & & & & 0,278 \\
\hline Item 37 & 0,500 & & & & 0,331 \\
\hline Item 46 & 0,496 & & & & 0,364 \\
\hline Item 7 & 0,425 & & & & 0,266 \\
\hline Item 31 & 0,413 & & & & 0,339 \\
\hline Item 38 & 0,398 & & & & 0,166 \\
\hline Item 33 & 0,397 & & & & 0,187 \\
\hline Item 47 & 0,339 & & & & 0,193 \\
\hline Item 4 & 0,328 & & & & 0,169 \\
\hline Item 20 & 0,271 & & & & 0,143 \\
\hline Item 41 & 0,086 & & & & 0,017 \\
\hline Item 24 & & 0,604 & & & 0,380 \\
\hline Item 22 & & 0,566 & & & 0,349 \\
\hline Item 6 & & 0,562 & & & 0,386 \\
\hline Item 39 & & 0,532 & & & 0,319 \\
\hline Item 16 & & 0,531 & & & 0,350 \\
\hline Item 45 & & 0,515 & & & 0,446 \\
\hline Item 21 & & 0,458 & & & 0,390 \\
\hline Item 13 & & 0,447 & & & 0,327 \\
\hline Item 26 & & 0,425 & & & 0,272 \\
\hline Item 14 & & 0,413 & & & 0,255 \\
\hline Item 19 & & 0,238 & & & 0,121 \\
\hline Item 48 & & $-0,475$ & & & 0,250 \\
\hline Item 11 & & & 0,641 & & 0,476 \\
\hline Item 18 & & & 0,629 & & 0,426 \\
\hline Item 28 & & & 0,539 & & 0,395 \\
\hline Item 32 & , & & 0,522 & & 0,353 \\
\hline Item 30 & & & 0,510 & & 0,317 \\
\hline Item 35 & & & 0,482 & & 0,325 \\
\hline Item 1 & & & 0,426 & & 0,214 \\
\hline Item 40 & & & 0,413 & & 0,324 \\
\hline Item 42 & & & 0,374 & & 0,225 \\
\hline Item 27 & & & 0,325 & & 0,165 \\
\hline Item 3 & & & 0,305 & & 0,170 \\
\hline Item 25 & & & 0,244 & & 0,068 \\
\hline Item 12 & & & $-0,529$ & & 0,306 \\
\hline Item 8 & & & & 0,505 & 0,484 \\
\hline Item 34 & & & & 0,461 & 0,258 \\
\hline Item 2 & & & & 0,416 & 0,199 \\
\hline Item 43 & & & & 0,408 & 0,266 \\
\hline Item 36 & & & & 0,399 & 0,317 \\
\hline Item 5 & & & & 0,159 & 0,062 \\
\hline
\end{tabular}

entre 0,132 a 0,196 . O fator 1 apresentou alfa de Cronbach de 0,764 seguido do fator 2 com 0,707.

Desse modo, decidiu-se náo excluir os itens contidos no fator, visto que, encontrou-se valor
Quadro 2. Correlação item-total, alfa de Cronbach e alfa de Cronbach se item deletado dos fatores e escore total do Instrumento do Questionário de Conforto Geral, utilizando-se 0 componente principal de análise

\begin{tabular}{|c|c|c|c|c|c|}
\hline & $\begin{array}{l}\text { Média de } \\
\text { escala se } \\
\text { o item for } \\
\text { excluído }\end{array}$ & $\begin{array}{c}\text { Variância de } \\
\text { escala se } \\
\text { o item for } \\
\text { excluído }\end{array}$ & $\begin{array}{l}\text { Correlação } \\
\text { de item total } \\
\text { corrigida }\end{array}$ & $\begin{array}{c}\text { Correlação } \\
\text { múltipla ao } \\
\text { quadrado }\end{array}$ & $\begin{array}{c}\text { Alfa de } \\
\text { Cronbach se } \\
\text { o item for } \\
\text { excluído }\end{array}$ \\
\hline Item 1 & 144,0750 & 266,070 & 0,251 & 0,333 & 0,818 \\
\hline Item 2 & 145,5042 & 269,615 & 0,147 & 0,306 & 0,821 \\
\hline Item 3 & 143,9792 & 267,702 & 0,220 & 0,265 & 0,819 \\
\hline Item 4 & 143,5750 & 272,798 & 0,121 & 0,267 & 0,820 \\
\hline Item 5 & 145,3792 & 273,165 & 0,051 & 0,200 & 0,824 \\
\hline Item 6 & 144,9875 & 255,862 & 0,465 & 0,385 & 0,811 \\
\hline Item 7 & 143,8792 & 263,287 & 0,393 & 0,361 & 0,814 \\
\hline Item 8 & 145,1250 & 259,089 & 0,372 & 0,454 & 0,814 \\
\hline Item 9 & 143,5167 & 267,180 & 0,429 & 0,555 & 0,815 \\
\hline Item 10 & 143,5542 & 269,436 & 0,279 & 0,518 & 0,817 \\
\hline Item 11 & 143,9375 & 263,214 & 0,397 & 0,494 & 0,814 \\
\hline Item 12 & 145,3708 & 286,912 & $-0,283$ & 0,411 & 0,834 \\
\hline Item 13 & 144,0417 & 261,237 & 0,417 & 0,448 & 0,813 \\
\hline Item 14 & 144,4167 & 259,809 & 0,392 & 0,343 & 0,814 \\
\hline Item 15 & 143,7292 & 266,993 & 0,353 & 0,362 & 0,816 \\
\hline Item 16 & 144,8667 & 257,212 & 0,389 & 0,353 & 0,813 \\
\hline Item 17 & 143,4417 & 272,925 & 0,180 & 0,286 & 0,819 \\
\hline Item 18 & 144,2458 & 261,651 & 0,354 & 0,485 & 0,815 \\
\hline Item 19 & 143,6417 & 268,984 & 0,228 & 0,234 & 0,818 \\
\hline Item 20 & 145,0042 & 263,736 & 0,285 & 0,316 & 0,817 \\
\hline Item 21 & 143,9792 & 257,954 & 0,501 & 0,456 & 0,811 \\
\hline Item 22 & 144,6083 & 257,553 & 0,407 & 0,454 & 0,813 \\
\hline Item 23 & 143,4542 & 270,935 & 0,276 & 0,402 & 0,818 \\
\hline Item 24 & 145,2958 & 260,803 & 0,365 & 0,420 & 0,814 \\
\hline Item 25 & 144,2708 & 268,466 & 0,144 & 0,169 & 0,822 \\
\hline Item 26 & 144,7125 & 261,712 & 0,285 & 0,347 & 0,817 \\
\hline Item 27 & 143,8583 & 270,741 & 0,153 & 0,187 & 0,820 \\
\hline Item 28 & 144,2250 & 256,493 & 0,471 & 0,400 & 0,811 \\
\hline Item 29 & 143,8375 & 264,840 & 0,371 & 0,368 & 0,815 \\
\hline Item 30 & 144,0375 & 267,534 & 0,236 & 0,335 & 0,818 \\
\hline Item 31 & 143,9250 & 260,061 & 0,492 & 0,426 & 0,812 \\
\hline Item 32 & 144,6333 & 265,062 & 0,242 & 0,366 & 0,818 \\
\hline Item 33 & 144,8083 & 266,951 & 0,246 & 0,305 & 0,818 \\
\hline Item 34 & 144,2833 & 270,798 & 0,128 & 0,291 & 0,821 \\
\hline Item 35 & 144,5375 & 257,589 & 0,419 & 0,375 & 0,813 \\
\hline Item 36 & 144,0792 & 260,374 & 0,390 & 0,443 & 0,814 \\
\hline Item 37 & 144,1250 & 262,235 & 0,365 & 0,391 & 0,815 \\
\hline Item 38 & 143,4417 & 271,988 & 0,231 & 0,331 & 0,819 \\
\hline Item 39 & 145,3250 & 267,727 & 0,181 & 0,294 & 0,820 \\
\hline Item 40 & 143,8750 & 261,340 & 0,459 & 0,401 & 0,813 \\
\hline Item 41 & 143,3833 & 275,635 & 0,088 & 0,142 & 0,820 \\
\hline Item 42 & 143,9500 & 269,219 & 0,174 & 0,305 & 0,820 \\
\hline Item 43 & 144,7750 & 279,020 & $-0,094$ & 0,247 & 0,829 \\
\hline Item 44 & 143,5083 & 266,954 & 0,452 & 0,445 & 0,815 \\
\hline Item 45 & 143,8583 & 258,365 & 0,551 & 0,512 & 0,810 \\
\hline Item 46 & 143,8750 & 268,210 & 0,258 & 0,358 & 0,818 \\
\hline Item 47 & 143,8125 & 265,927 & 0,318 & 0,351 & 0,816 \\
\hline Item 48 & 143,7667 & 285,811 & $-0,339$ & 0,366 & 0,830 \\
\hline
\end{tabular}

satisfatório do alfa de Cronbach geral. Constatouse ainda que os valores estimados pelo coeficiente alfa de Cronbach para os fatores não se alteraram se itens deletados, exceto para o fator 1 , no qual se 
constatou redução no alfa (de 0,764 para 0,702$)$ se deletado o item 31.

Assim, constata-se que permaneceram 33 itens entre as versôes validadas entre o grupo de especialistas e a análise fatorial exploratória respeitando os princípios psicométricos com perda de 15 itens (3, $4,5,6,7,18,19,20,22,24,25,27,33,35,36$, $39,41,42,47)$.

\section{Discussão}

Assim, reporta-se como desejável que esse instrumento seja aplicado em amostras com culturas e crenças diferentes para avançar no seu desenvolvimento e trazer mais evidências para fortalecer as análises de consistência interna e de dimensionalidade da estrutura fatorial. Destaca-se, portanto, a limitação quanto a comparação dos resultados desta análise com pesquisas de mesmo propósito em outras culturas e países, visto não terem sido encontradas validaçôes do instrumento por meio de análise fatorial exploratória e confirmatória.

Os resultados alcançados no estudo de confiabilidade da versão brasileira do GCQ para pacientes renais crônicos apresentaram algumas divergências quanto aos parâmetros publicados para a versão original, ${ }^{(2)}$ referindo-se neste caso, a realocação de itens dentro dos domínios, ou a sua exclusão. Essa possibilidade é previsível, tendo em vista que o constructo em análise é permeado por subjetividades próprias do sujeito e do contexto social e cultural em que este convive, assim, além de considerar aspectos relativos aos dados objetivos e concretos da doença, é fundamental atribuir relevância ao processo saúde-doença e a dinâmica de interação da pessoa com o momento e o meio em que vive. ${ }^{(11)}$

A consistência interna e validade do instrumento foram evidenciadas pela mensuração do alfa de Cronbach geral de 0,805 o que permite alinhá-lo a outros estudos de validação e análise psicométrica de questionários voltados a análise do mesmo constructo com populações distintas, como por exemplo: 0,98 e 0,97 com pacientes em final de vida e seus cuidadores diretos, respectivamente, em Ohio; ${ }^{(15)}$ 0,795 em cuidadores de pessoas com doença crônica avançada em Portugal; ${ }^{(16)} 0,923$ no contexto de adoecimento em situaçóes críticas, no Brasil; ${ }^{(17)}$ e, 0,769 em uma versão curta validada na Indonésia para pacientes em hemodiálise. ${ }^{(18)}$

Observa-se, contudo, que há diferenças consideráveis entre estes resultados e aqueles apresentados na adaptação transcultural para a realidade brasileira realizada por juízes, em que a equivalência geral do instrumento foi de 0,943 com alfa de Cronbach igual a 0,8 , sem perdas ou realocaçóes de itens. ${ }^{(6)} \mathrm{A}$ estrutura fatorial exploratória confirmou a presença de quatro fatores (psicoespiritual; sociocultural; ambiental e físico) conforme apresentado pelo instrumento original como domínios. ${ }^{(2)}$ No entanto, vale ressaltar que a análise da comunalidade dos itens revelou a possibilidade de que alguns deles não são explicados pelos fatores ora atribuídos, sugerindo assim, uma contribuição pequena do item ao modelo construído. $^{(19)}$

$\mathrm{O}$ fato de o teste de esfericidade de Bartlett ser estatisticamente significativo e que o valor da $\mathrm{KMO}$ estar acima de 0,50 indicou que o tamanho da amostra eram estatisticamente significantes para a análise fatorial.

Como resultado da análise fatorial exploratória, os itens do questionário foram agrupados em 10 subdimensóes. O fato do QGC de 33 itens consistir em 10 dimensôes mostra que algumas dimensões não contêm itens suficientes. A análise do gráfico de autovalor mostrou que a aceleração da queda após o quarto fator era baixa sugere que o modelo de quatro fatores seria apropriado para o questionário de 33 itens. O modelo de quatro fatores responde por $38,01 \%$ da variância total da variação total.

$\mathrm{Na}$ literatura, afirma-se que a variância explicada deve estar entre 40,0 e 60,0\%. ${ }^{(20)}$ No entanto, em estudos de sociologia e psicológicos afirma-se que, se a variância explicada for superior a 35\%, seria suficiente. ${ }^{(18)}$ Assim, visto que o QGC mede conforto, um conceito abstrato e que a variação explicada é menor que $40,0 \%$ é uma situação tangível. ${ }^{(21,22)}$

Verifica-se que na análise das propriedades psicométricas do Nurse Comfort Questionnaire construído na Turquia, o scree plot apresentou resultados apenas para 3 fatores com os 39 itens com variabilidade de $37.87 \%{ }^{(20)}$ demonstrando que um 
tratamento estatístico mais robusto permite extrair de forma mais precisa a avaliação acerca do constructo conforto sem perder a essência dos seus atributos principais. Constatou-se ainda nesse estudo que os valores alfa de Cronbach das subdimensões foram de 0,859 para o primeiro fator, 0,846 para o segundo fator e 0,818 para o terceiro fator. Nesse estudo, verificou-se ampla variabilidade entre os quatros fatores desde 0,327 até 0,764.

Defende-se que a análise das características psicométricas deste instrumento seja realizada na medida em que sua tradução/adaptação transcultural aconteça, ${ }^{(23)}$ visto que este procedimento garante aos pesquisadores e profissionais de saúde um instrumento técnica e cientificamente validado para qualificar o cuidado, além de garantir a eficácia na medida adequada do construto para fins de tomada de decisão, dadas as suas limitaçóes quanto a reprodutibilidade e interpretação em diferentes culturas e condiçôes de adoecimento.

\section{Conclusão}

As análises estatísticas realizadas permitiram concluir que o QCG - Versão Brasileira, possui evidências de validade psicométrica com base na estrutura interna, demonstrando ser considerado confiável e válido para medir o conforto em pacientes renais crônicos em tratamento hemodialítico. Assim, este material vem a fomentar uma avaliação mais criteriosa e completa do conforto no público alvo, permeando os domínios do conforto a ser utilizado com segurança por enfermeiros para nortear a implementação de intervençóes ativas afim de garantir um melhor conforto.

\section{Colaborações}

Melo GAA, Silva RA, Pereira FG, Lima LA, Magalhães TM, Silva VM e Caetano JÁ contribuíram com a concepção do estudo, análise e interpretação dos dados, redação do artigo, revisão crítica relevante do conteúdo intelectual e aprovação da versão final a ser publicada.

\section{Referências}

1. Ponte KM, Silva LF. Comfort as a result of nursing care: an integrative review. J Res Fundam Care. 2015;7(2):2603-14.

2. thecomfortline.com [Internet] San Diego: Comfort Line; c2019 [cited 2019 Aug 30]. Available from: http://thecomfortline.com/

3. Tosun B, Aslan Ö, Tunay S, Akyüz A, Özkan H, Bek D, et al. Turkish Version of Kolcaba's Immobilization Comfort Questionnaire: A Validity and Reliability Study. Asian Nurs Res (Korean Soc Nurs Sci). 2015;9(4):278-84.

4. Pinto SM, Berenguer SM, Martins JC, Kolcaba K. Cultural adaptation and validation of the Portuguese End of Life Spiritual Comfort Questionnaire in Palliative Care patients. Porto Biomed J. 2016;1(4):147-52.

5. Góis JÁ, Freitas KS, Kolcaba K, Mussi FC. Cross-cultural adaptation of the General Comfort Questionnaire to Brazilian patients with myocardial infarction. Rev Bras Enferm. 2018;71(6):2998-3005.

6. Melo GA, Silva RA, Pereira FG, Caetano JA. Cultural adaptation and reliability of the General Comfort Questionnaire for chronic renal patients in Brazil. Rev Lat Am Enfermagem. 2017;25(0):e2963.

7. Melo GA, Silva RA, Aguiar LL, Pereira FG, Galindo Neto NM, Caetano JÁ. Content validation of the Brazilian version General Comfort Questionnaire. Rev Rene. 2019;20:e41788.

8. Estridge KM, Morris DL, Kolcaba K, Winkelman C. Comfort and fluid retention in adult patients receiving hemodialysis. Nephrol Nurs J. 2018;45(1):25-60.

9. Melo GA, Aguiar LL, Silva RA, Quirino GD, Pinheiro AK, Caetano JA. Factors related to impaired comfort in chronic kidney disease patients on hemodialysis. Rev Bras Enferm. 2019;72(4):889-95.

10. Beser A, Bahar Z, Kissal A, Cal A, Cavusoglu F, Mert H, et al. Psychometric properties of the Turkish version of the TuberculosisRelated Stigma scale. Acta Paul Enferm. 2018;31(4):374-81.

11. Souza DJ, Oliveira LR, Lemos RCA, Felix MMS, D'Innocenzo M. Validade de construto do Índice de Humanização dos Serviços de Saúde. Cogitare Enferm. 2016; 21(4):1-9.

12. Stacciarini TS, Pace AE. Confirmatory factor analysis of the Appraisal of SelfCare Agency Scale - Revised. Rev Lat Am Enfermagem. 2017;25(0):e2856.

13. Umann J, Silva RM, Kimura CA, Lautert L. Aplicações da modelagem de equações estruturais na enfermagem: revisão integrativa. Rev Eletr Enferm. 2017;19:a51.

14. Silva TO, Alves LB, Balieiro MM, Mandetta MA, Tanner A, Shields L. Cross-cultural adaptation of an instrument to measure the familycentered care. Acta Paul Enferm. 2015;28(2):107-12.

15. Novak B, Kolcaba K, Steiner R, Dowd T. Measuring comfort in caregivers and patients during late end-of-life care. Am J Hosp Palliat Care. 2001;18(3):170-80.

16. Marques RM, Dixe MA, Querido Al, Sousa PP. Revalidation of the Holistic Comfort Questionnaire - Family for caregivers of people with advanced chronic disease. Rev Ref. 2016;4(11):91-100.

17. Freitas KS, Menezes IG, Mussi FC. Validation of the Comfort scale for relatives of people in critical states of health. Rev Lat Am Enfermagem. 2015;23(4):660-8.

18. Artanti ER, Nurjannah I, Subroto S. Subroto. Validity and reliability of shortened General Comfort Questionnaire in Indonesian Version. Belitung Nurs J. 2018;4(4):366-72.

19. Pedrosa RB, Rodrigues RC, Padilha KM, Gallani MC, Alexandre NM. Factor analysis of an instrument to measure the impact of disease on daily life. Rev Bras Enferm. 2016;69(4):697-704. 
20. Cinar Yucel ş, Goke Arslan G, Ergin E, Kuguoglu S. Psychometric Characteristics of the Turkish Version of the Nurse Comfort Questionnaire. J Relig Health. 2019;58(5):1803-16.

21. Yaşıı̆lu MM. Sosyal bilimlerde faktör analizi ve geçerlilik: keşfedici ve doğrulayıcı faktör analizlerinin kullanılması. Istanbul Bus Res 2017;46:74-85.
22. Ferrandiz EF, Martín-Baena D. Translation and validation of a Spanish version of the Kolcaba's general comfort questionnaire in hospital nurses. Int J Nurs (N Y). 2015;2(1):113-9.

23. Bektas M, Bektas I, Selekoğlu Y, Kudubes AA, Altan SS, Ayar D. Psychometric properties of the Turkish version of the Emotional Eating Scale for children and adolescents. Eat Behav. 2016;22:217-21.

\section{General Comfort Questionnaire, versão Brasileira}

Itens do Instrumento do Questionário de Conforto Geral que permaneceram após a análise fatorial exploratória.

\begin{tabular}{|c|c|c|c|c|}
\hline 1. Sinto meu corpo relaxado agora & 4 & 3 & 2 & 1 \\
\hline 2. Eu me sinto útil porque estou trabalhando muito & 4 & 3 & 2 & 1 \\
\hline 8. Eu me sinto dependente dos outros & 4 & 3 & 2 & 1 \\
\hline 9. Eu sinto que minha vida vale a pena & 4 & 3 & 2 & 1 \\
\hline 10. Eu me sinto satisfeito(a) por saber que eu sou amado(a) & 4 & 3 & 2 & 1 \\
\hline 11. Estes ambientes são agradáveis & 4 & 3 & 2 & 1 \\
\hline 12. 0 barulho não me deixa descansar & 4 & 3 & 2 & 1 \\
\hline 13. Ninguém me entende & 4 & 3 & 2 & 1 \\
\hline 14. Minha dor é difícil de ser suportada & 4 & 3 & 2 & 1 \\
\hline 15. Eu estou motivado(a) em fazer o meu melhor & 4 & 3 & 2 & 1 \\
\hline 16. Eu fico triste quando estou sozinho(a) & 4 & 3 & 2 & 1 \\
\hline 17. Minha fé me ajuda a não ter medo & 4 & 3 & 2 & 1 \\
\hline 21. Este ambiente me faz sentir medo & 4 & 3 & 2 & 1 \\
\hline 23. Eu tenho uma pessoa(s) que me faz (em) sentir cuidado (a) & 4 & 3 & 2 & 1 \\
\hline 26. Eu gostaria de ver meu médico com mais frequência & 4 & 3 & 2 & 1 \\
\hline 28. Eu estou muito cansado (a) & 4 & 3 & 2 & 1 \\
\hline 29. Eu posso superar minha dor & 4 & 3 & 2 & 1 \\
\hline 30. 0 humor daqui me faz sentir melhor & 4 & 3 & 2 & 1 \\
\hline 31. Eu estou contente & 4 & 3 & 2 & 1 \\
\hline 32. Esta cadeira (cama) me machuca & 4 & 3 & 2 & 1 \\
\hline 34. Meus pertences não estão aqui & 4 & 3 & 2 & 1 \\
\hline 37. Meus amigos lembram-se de mim com mensagens e telefonemas & 4 & 3 & 2 & 1 \\
\hline 38. Minhas crenças me dão paz de espírito & 4 & 3 & 2 & 1 \\
\hline 40. Eu me sinto fora de controle & 4 & 3 & 2 & 1 \\
\hline 43. Eu estou sozinho (a), mas não solitário (a) & 4 & 3 & 2 & 1 \\
\hline 44. Eu me sinto em paz & 4 & 3 & 2 & 1 \\
\hline 45. Eu estou deprimido (a) & 4 & 3 & 2 & 1 \\
\hline 46. Eu tenho encontrado sentido na minha vida & 4 & 3 & 2 & 1 \\
\hline 48. Eu preciso me sentir bem novamente & 4 & 3 & 2 & 1 \\
\hline
\end{tabular}

4: Concordo Totalmente; 3: Concordo; 2: Discordo; 1: Discordo Totalmente 